\title{
A Rare Cause of Lower Abdomen Pain - Primary Urinary Bladder Amyloidosis
}

\author{
Kalpesh Parmar*, Abhishek Thakur, Kumar Rajiv Ranjan and Santosh Kumar \\ Department of Urology, PGIMER, Chandigarh
}

\begin{abstract}
Primary urinary bladder amyloidosis is a rare disease. Its importance lies in the ability of the disease to masquerade the carcinoma urinary bladder in clinical presentation and imaging but with a distinctively contrasting treatment. The commonest presentation is painless hematuria. Definitive diagnosis is made on histopathology and by ruling out systemic amyloidosis. Here, we report a 60 -year-old male presenting with lower abdomen pain diagnosed with primary urinary bladder amyloidosis and discuss the management based on current literature.
\end{abstract}

Keywords: Amyloidosis, primary, haematuria, TURBT

\section{Introduction}

Amyloidosis is typified by the deposition of a hyaline, eosinophilic protein like material in the various organs. It can be primary (AL Amyloidosis) or secondary (AA Amyloidosis). Secondary amyloidosis may be a complication of long term inflammatory disease or long term inflammation but primary amyloidosis occurs due to plasma cell disorders producing excess monoclonal immunoglobulin light chain in bone marrow and hence profoundly found in blood and urine. Primary amyloidosis occurs with lymphoma, multiple myeloma and Waldenstrom's macroglobulinemia. It may involve multiple organs. Various organ involvements seen are gastrointestinal tract, muscular tissue, adipose tissue and genitourinary tract. Localized involvement of genitourinary tract is uncommon. The recent available data quotes only 200 reported cases of localised primary urinary bladder amyloidosis [1]. Classical presentation of urinary bladder amyloidosis is haematuria. So, it may imitate urinary bladder malignancy and even radiologic and cystoscopy features are indistinguishable. Definitive diagnosis is by histopathology and immunohistochemistry. Systemic involvement should be ruled out.

\section{Case report}

A 60-year male, chronic smoker and hypertensive, presented with lower abdomen pain of 6 months duration. The pain was dull aching, mild in intensity, intermittent and relieved on taking analgesics. There was no history of haematuria, lower urinary tract symptoms, loss of weight or appetite. His past medical and family history was insignificant. On examination, he was conscious, alert, average built and vitals were stable. Systemic examination was grossly normal. His haemoglobin was $11.3 \mathrm{gm} / \mathrm{dl}$, serum creatinine was $1.1 \mathrm{mg} / \mathrm{dl}$, INR was 0.9 , urine analysis showed 4-6 pus cells and culture was sterile. Ultrasound abdomen reported echogenic mass in the urinary bladder. Contrast enhanced computed tomography revealed heterogenous mass of $3 * 2$ $\mathrm{cm}$ in the right lateral wall of urinary bladder. (Figure 1) Rest of the solid organs were normal and there were no enlarged retroperitoneal lymph nodes. Patient was counselled for transurethral resection of bladder tumour (TURBT). After written informed consent, patient was operated under regional anaesthesia. Intraoperatively, there was single papillary growth in right posterolateral wall of urinary bladder just above the ureteric orifice with surrounding erythema. (Figure 2) Using 26 French resectoscope and $30^{\circ}$ lens, complete resection of mass was done and send for histopathology. Postoperative recovery was uneventful. Histopathology report showed multinucleate giant cells with foamy macrophages and pale acellular material. There was no evidence of malignant cells. Immunostaining showed strong positivity for Congo red staining and apple green birefringence suggestive of amyloidosis AL type (light chain). (Figure 3) Systemic investigations were carried out to rule out systemic amyloidosis. The urinary Bence jones proteins and 24 hour urine protein electrophoresis were negative. Abdominal fat pad and sigmoid colon biopsy was also negative. Patient was asymptomatic at 3 months follow up and repeat cystoscopy showed only scar of previous surgery. At 2 years follow up, patient is disease free on cystoscopy and doing well.

\section{Discussion}

Amyloidosis is a condition of protein metabolism typified by extracellular deposition of abnormal protein fibrils. The term was coined by Virchow in 1854 meaning 'starch-like' to illustrate tissue deposits that tainted with iodine solutions [2]. Primary amyloidosis is taught to be an acquired plasma 


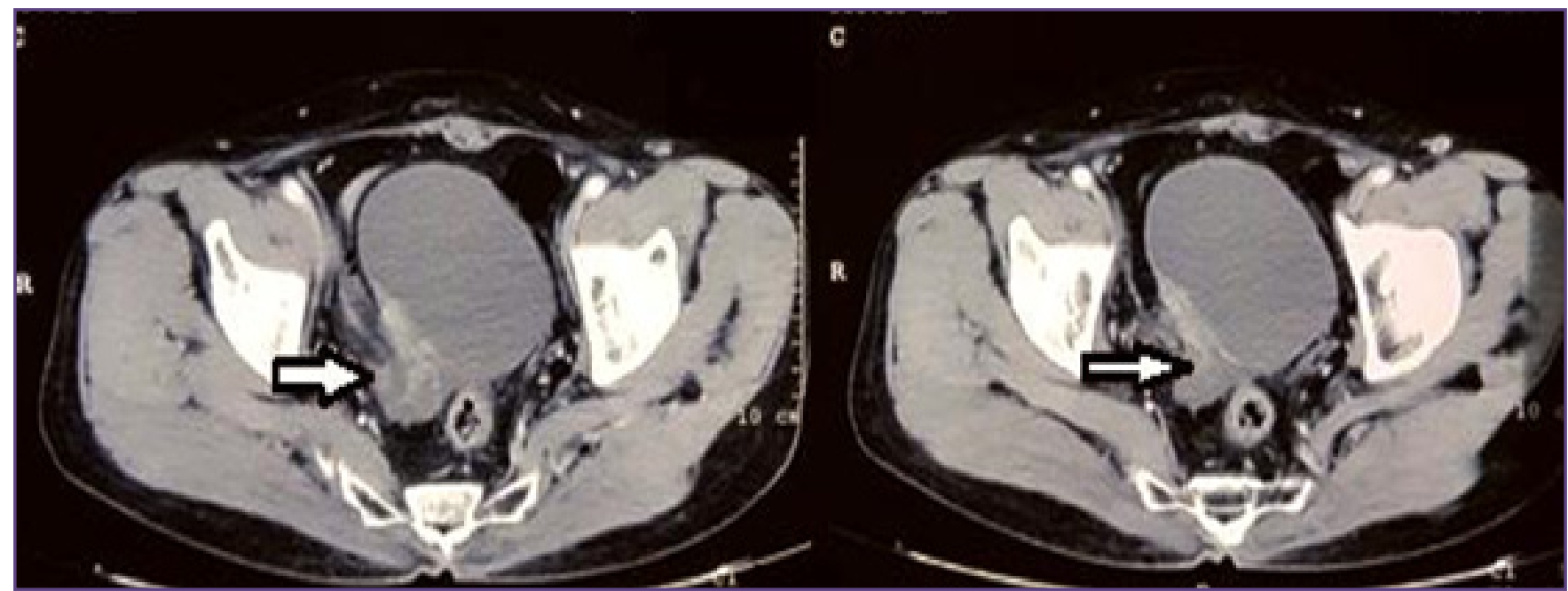

Fig. 1: Contrast enhanced computed tomography (CECT) abdomen axial images showing right posterolateral wall thickening with enhancement and loss of fat planes with seminal vesicles and extension into peri vesical tissues.

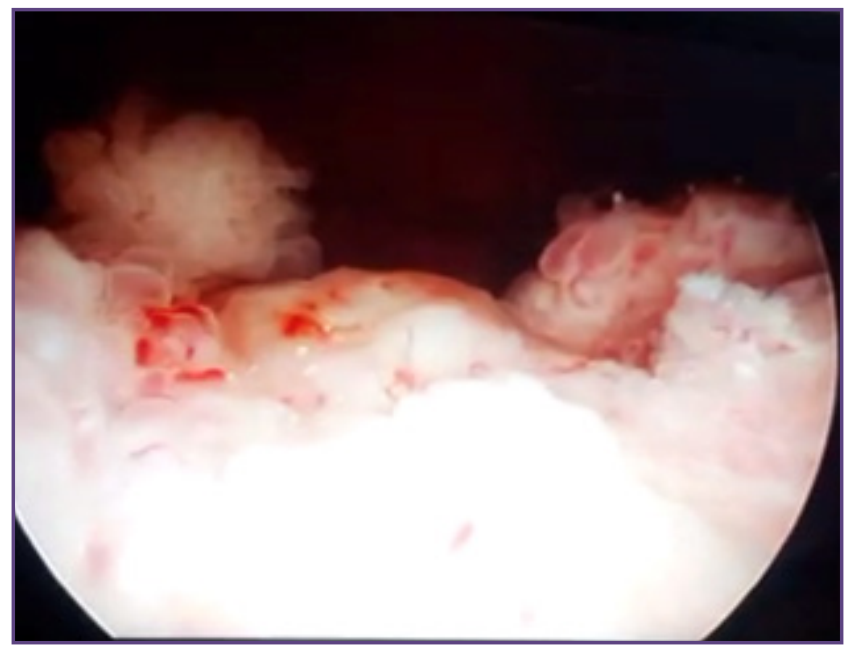

Fig. 2: Intraoperative image showing papillary growth with surrounding erythema in right posterolateral wall of urinary bladder.

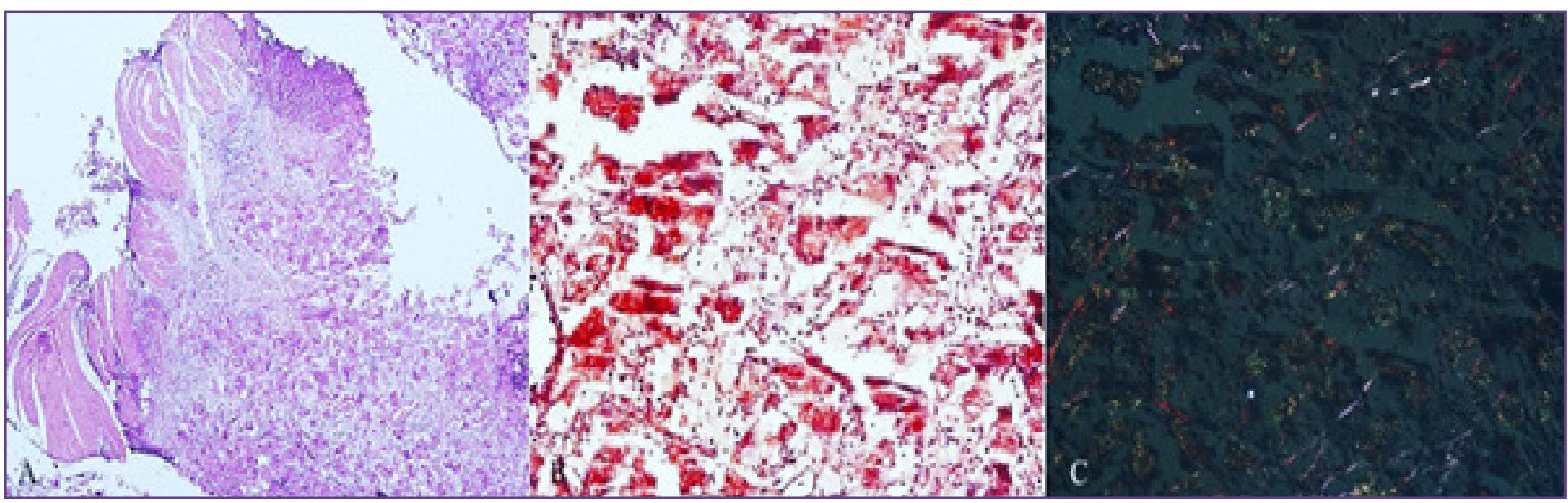

Fig 3: Histopathology image showing (A) multinucleate giant cells with foamy macrophages with pale acellular material (H \& E x10 ) (B) dense Congo red positivity (Congo red stain) (C) apple green birefringence ( polarized light chain imaging). 
cell disorder in which a monoclonal immunoglobulin light chain is produced in the bone marrow and frequently found in the blood or urine. Secondary amyloidosis is caused by chronic inflammation and systemic diseases like rheumatoid arthritis, familial Mediterranean fever, osteomyelitis, Crohn's disease etc. It is usually a systemic disease, however rarely it may be localised to one organ. About 6-9\% of all cases of amyloidosis are confined to one organ system. The most commonly involved systems are respiratory tract, skin and genitourinary tracts ${ }^{[3]}$. In the genitourinary tract, deposition can occur anywhere from kidney to urinary bladder, urethra and even penis. Renal involvement is virtually always seen in secondary amyloidosis and in nearly $50 \%$ of the cases of primary amyloidosis. This is in contrast to the urinary bladder, which is mostly affected in primary localized amyloidosis. Primary amyloidosis of urinary bladder is linked to chronic inflammation and cystitis. The inflow of lymphoplasmacytic cells into the submucosa of the bladder transform into monoclonal cells that secrete an abnormal light chain type immunoglobulin. Bladder amyloid classically presents with visible haematuria and storage bladder symptoms, mimicking bladder malignancy. Tirzaman et al reported gross hematuria in $77 \%$ cases and irritative voiding symptoms in $23 \%$ in his series of 31 cases ${ }^{[4]}$. In the index case, patient presented with vague abdomen pain only. Primary bladder amyloidosis has a male preponderance and usually occurs at a mean age of 50-70 years. The amyloid deposition is found predominantly in the posterior bladder wall and the trigone for reasons unknown. In this case, amyloid was present in right lateral wall of urinary bladder. Amyloidosis of the bladder presents a great task to the urologist because of its close resemblance with an infiltrating neoplasm of urinary bladder. CT and MR imaging may depict focal or, less commonly, diffuse thickening of the bladder wall or a filling defect in the bladder lumen ${ }^{[5]}$. Cystoscopy evaluation is important but often appearances are arduous to construe with a variation of appearances ranging from ulcerated masses to diffuse thickening. Typically, in localized amyloidosis of the bladder, amyloid deposits are demonstrated in the lamina propria and muscularis propria with vessel wall involvement. Transurethral resection of the lesion and histopathological examination are important to establish a diagnosis and exclude malignancy. Presence of apple-green birefringence on Congo red staining when viewed with polarised light is classical of amyloidosis. It is important to rule out systemic involvement in essentially all $\operatorname{cases}^{[6]}$. Subsequent follow up with cystoscopy is recommended due to a reported recurrence rate of $50 \%$. Salahia et al reported early recurrence at 4 months following TURBT for primary urinary bladder amyloidosis which required re-resection ${ }^{[5]}$. Intravesical administration of dimethyl sulphoxide or the use of oral colchicine have been reported in literature for preventing recurrence; however, their clinical efficacy remains limited ${ }^{[6]}$. Rarely it may be associated with high grade transitional cell cancer and need aggressive procedures like cystectomy in lieu of incessant symptoms or life threatening haemorrhage. In the index case, patient had no recurrence on follow up and doing well.

\section{Conclusion}

Primary urinary bladder amyloidosis is scarce. Lower abdomen pain is unusual presentation. While malignancy is always the concern, transurethral resection and histopathology examination is essential for diagnosis. Systemic involvement should be ruled out in all cases. Long term follow up with yearly cystoscopy is recommended.

\section{Acknowledgement}

We sincerely thank the patient for being cooperative during hospital stay and treatment

\section{Funding}

None

\section{Competing interest}

None

\section{References}

1. Wilkinson M, Fanning DM, Flood H. Primary bladder amyloidosis. BMJ Case Rep. a2011 Jul 20:2011.

2. Jain M, Kumari N, Chhabra P, Gupta RK. Localized amyloidosis of urinary bladder: a diagnostic dilemma.Indian J Pathol Microbiol. 2008; 51(2) : 247 -9

3. Isoglu CS, Turk H, Karabicak M, et al. Cases of a Borderline Pathology That Can Mimic Bladder Cancer: Primary Amyloidosis of Urinary Bladder. J Urolog Surg 2015;2:94-6.

4. Tirzaman O, Wahner-Roedler DL, Malek RS, et al. Primary localized amyloidosis of the urinary bladder. Mayo Clin Proc 2000;75:1264-8.

5. Salahia MG, Kebbe Y, Arora A, et al. Primary bladder amylodoisis mimicking bladder malignancy: A case report and literature review. Urol Nephrol Open Access J 2014;1(2):1-4.

6. Gupta P, Hanamshetti S, Kulkarni JN. Primary amyloidosis with high grade transitional cell carcinoma of bladder: A rare case report. J Can Res Ther 2012;8:297-9.

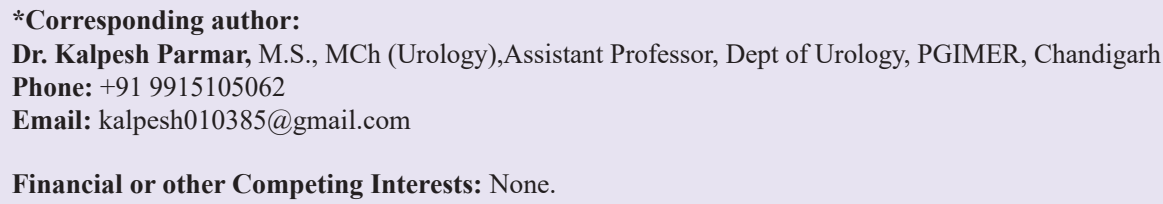

Date of Submission : 21/09/2020

Date of Final Revision : 04/01/2021

Date of Acceptance $\quad: 08 / 01 / 2021$

Date of Publication : $\quad 30 / 03 / 2021$ 